\title{
Droht der Europäischen Währungsunion eine "Japanisierung"?
}

Leef H. Dierks

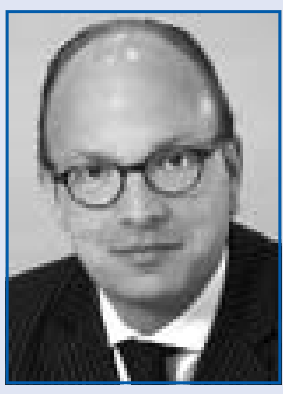

Prof. Dr. Leef H. Dierks ist Inhaber der Professur für Allgemeine Betriebswirtschaftslehre, insbes. Finanzierung und Internationale Kapitalmärkte an der Fachhochschule Lübeck. Bevorzugte Forschungsgebiete: Aktuelle Geldpolitik der EZB und ihre Auswirkungen auf Inflation und Deflation in Europa, Konsequenzen der Europäischen Bankenunion, Unterschiede der Finanzierung kleinerer und mittelständischer Unternehmen (KMU) in Europa.

Dieser Beitrag untersucht Ursachen und Auswirkungen der Deflation und hebt Parallelen zwischen der aktuellen Geld-, Fiskal- und Wirtschaftspolitik der Europäischen Währungsunion und jener des sogenannten Verlorenen Jahrzehnts in Japan hervor. Ungeachtet zahlreicher Ähnlichkeiten der Ausgangslage gelangt dieser Beitrag zu dem Ergebnis, dass die Gefahr einer „Japanisierung" der Europäischen Währungsunion, d. h. einer Periode fallender Preise bei rückläufiger Wirtschaftsleistung, in einzelnen Mitgliedsstaaten wie Spanien und Griechenland zwar bereits zu beobachten ist; für die Eurozone insgesamt jedoch aufgrund der Heterogenität der Volkswirtschaften nicht überbewertet werden sollte.

Stichwörter: Deflation, Geldpolitik, Finanzkrise, Eurozone, Japan

\section{Zahlreiche Parallelen zwischen Japan und der Eurozone}

Der aktuelle Zustand der Volkswirtschaften der Europäischen Währungsunion (EWU) weist zahlreiche Parallelen zu der Entwicklung Japans im sogenannten Verlorenen Jahrzehnt, d. h. den 1990-er Jahren, auf. Vor dem Hintergrund einer stetig sinkenden Inflationsrate, im November 2014 auf nur noch $0,3 \%$ p.a. und damit deutlich unterhalb des von der Europäischen Zentralbank (EZB) avisierten Zielwerts von ,unter aber nahe“ 2,00\% p.a. sowie einem Rückgang des Bruttoinlandsprodukts (BIP) von 0,4\% p.a. 2013 nach einem Rückgang von bereits 0,6 \% p.a. 2012, mehrten sich zuletzt die vor einer Deflation im Euroraum warnenden Stimmen (vgl. Kennedy, 2014). Neben einem infolge mehrerer Krisen derzeit verhaltenen Wirtschaftswachstum und einer Zurückhaltung der Banken bei der Kreditvergabe, gilt es in diesem Kontext insbesondere einen steigenden Wechselkurs sowie Ähnlichkeiten in der Geldpolitik, konkret also das Absenken des Hauptrefinanzierungszinssatzes auf ein historisches Tief, sowie die schleichende Erosion der langfristigen Inflationserwartungen hervorzuheben.

Doch lässt sich aus diesen teilweise frappierenden Ähnlichkeiten zwischen der Lage Japans in den 1990-er Jahren, dessen anhaltend deflationäre Tendenzen über Jahre hinweg als volkswirtschaftliche Anomalie (vgl. Abb. 1), vor der andere Industrienationen immun seien, galten, und der des heutigen Euroraums zwingend auf eine unmittelbar bevorstehende Deflation schließen?

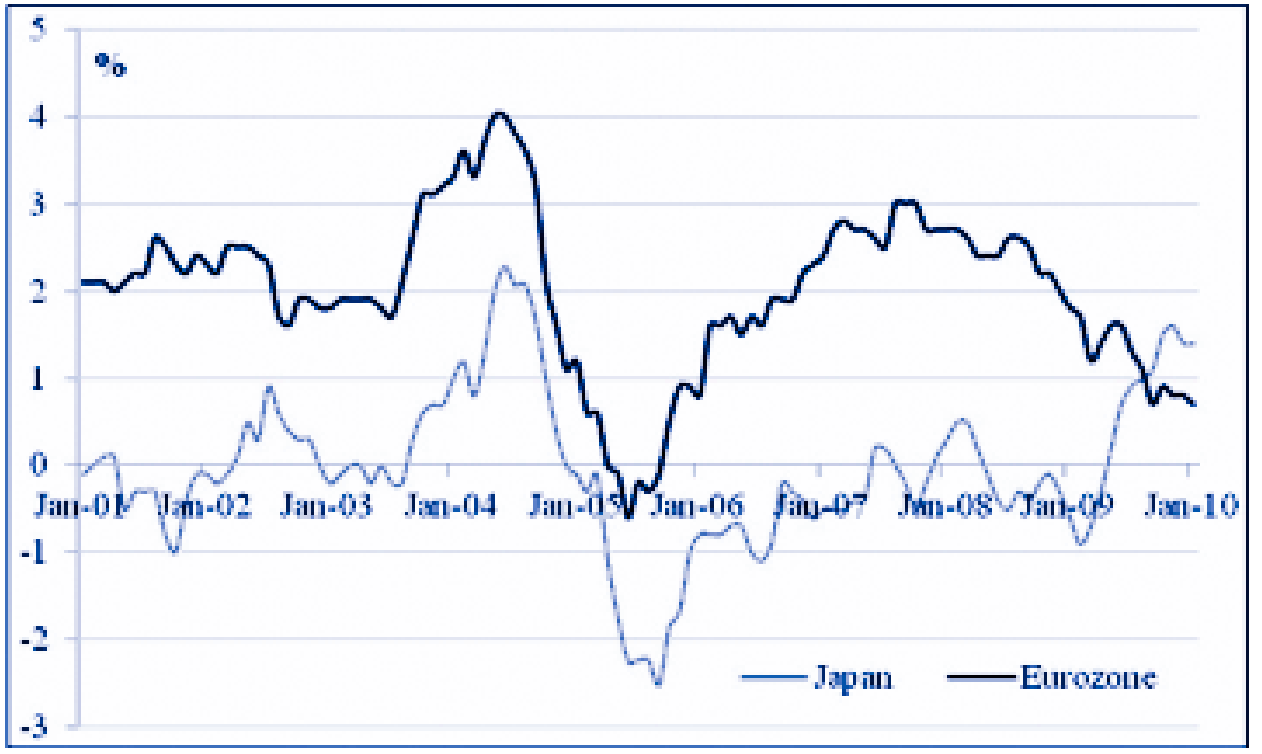

Quelle: Statistics Bureau of Japan (2014), EZB (2014)

Abb. 1: Entwicklung (in \%) der Verbraucherpreise in Japan und der Eurozone (2005-2014) 


\section{Deflation und Disinflation}

Deflation ist gemeinhin als allgemeiner, signifikanter und anhaltender Rückgang des Preisniveaus für Waren und Dienstleistungen, so beispielsweise des Verbraucherpreisindex oder des BIP-Deflators, also des Preisindex, der den Anstieg des Bruttoinlandsprodukts angibt, der auf eine Erhöhung der Preise zurückzuführen ist, definiert. Üblicherweise ist im Falle einer Deflation, die in der Regel zusammen mit einer wirtschaftlichen Depression auftritt, die gesamtwirtschaftliche Nachfrage geringer als das gesamtwirtschaftliche Angebot, d. h. die Geldmenge erscheint zu gering.

Während ein oder zwei Quartale fallender Preise formell zwar ebenfalls eine Deflation darstellen, ist eine derartige Entwicklung kaum besorgniserregend. Gleichwohl sollten selbst die Auswirkungen einer kurzfristigen Deflation nicht unterschätzt werden; schon diese führt u. a. zu ökonomischen Unsicherheiten, einer verzerrten Ressourcenallokation, Anreiz- und Verteilungsproblemen sowie einem unterdurchschnittlichen Wirtschaftswachstum.

Im Gegensatz zur Deflation versteht man unter Disinflation eine Verringerung des Preisniveauanstiegs, d. h. die Geschwindigkeit von Preissteigerungen nimmt ab. Somit ist Disinflation eine Verminderung der Inflation, jedoch kein Sinken des Preisniveaus.

\section{Determinanten der Deflation}

Einsetzen und Fortdauern einer Deflation ist in der Regel auf eine Vielzahl verschiedener Einflussfaktoren zurückzuführen. Betrachtet werde zunächst eine im Marktgleichgewicht befindliche Volkswirtschaft bei Vollbeschäftigung $\left(\pi^{*}, \mathrm{Y}^{*}\right)$, welches sich aus dem Schnittpunkt der aggregierten Nachfrage (AD) und dem aggregierten Angebot (AS) herleiten lässt (vgl. Abb. 2). Ein hinreichend großer, negativer Nachfragschock, der die Nachfragekurve von AD auf AD' verschiebt, vermag die Volkswirtschaft in eine deflationäre Region ( $\left.\pi^{* *}, \mathrm{Y}^{* *}\right)$ mit fallenden Preisen und geringerem Output zu bringen. Ein solcher externer
Schock könnte beispielsweise auf einen zyklischen Abschwung, das Platzen einer spekulativen Blase oder aber eine exzessiv restriktive Geld- bzw. Fiskalpolitik zurückgehen und den ursprünglichen deflationären Impuls durch einen Rückgang des Verbrauchervertrauens sowie der Erwartung fallender Preise verstärken. Alternativ ließe sich anhand eines positiven Angebotsschocks, der das aggregierte Angebot von AS auf AS', also in eine Region wachsendes Outputs trotz fallender Preise $\left(\pi^{\prime}, \mathrm{Y}^{\prime}\right)$, verschiebt, argumentieren. Positive Angebotsschocks lassen sich u. a. durch technologischen Fortschritt, eine zunehmende Produktivität, den Abbau von Handelshemmnissen oder einem Anstieg des Verbrauchervertrauens begründen. Denkbar wäre zudem eine Kombination aus Angebotsund Nachfrageschocks, die einerseits zwar den deflationären Druck erhöhen, andererseits aber die Auswirkungen auf den Output bessern $\left(\pi^{\mathrm{C}}, \mathrm{Y}^{\mathrm{C}}\right)$.

Sobald die Preise zu fallen beginnen, insbesondere jedoch im Falle eines Nachfrageschocks, steigt das Risiko einer sogenannten adversen Dynamik, d. h. der Gefahr einer sich selbst verstärkenden Spirale aus sinkenden Preisen und wirtschaftlichem Niedergang. Infolge eines solchen Schocks und bei Einsetzen einer Deflation wird der Nominalzins voraussichtlich sinken. Da dieser Null in der Regel jedoch nicht zu unterschreiten vermag, wird der Realzins zunächst positiv bleiben. Generell gilt, dass der Realzins umso höher ausfällt, je stärker die Deflation ausgeprägt ist. Die Geldpolitik wird in diesem Szenario nahezu bedeutungslos, da die Nachfrage nach Geld bei einem Zinssatz von oder aber nahe bei Null mehr oder weniger unendlich elastisch ist. Im Rahmen des klassischen IS-LM Ansatzes bedeutet dies, dass sofern die IS-Kurve die LM-Kurve in dem Bereich der flachen Steigung schneidet, ein Anstieg des Geldangebots die LM-Kurve zwar nach rechts auf LM' verschöbe, gleichwohl keinerlei Einfluss auf Zinsen oder Output hätte (vgl. Abb. 3). Dies ist die klassische Liquiditätsfalle.

Aus intertemporaler Perspektive erweist sich die Liquiditätsfalle aufgrund stabiler Erwartungen bei nominalen Rigiditäten als durchaus beständig. Solange die Marktteil-

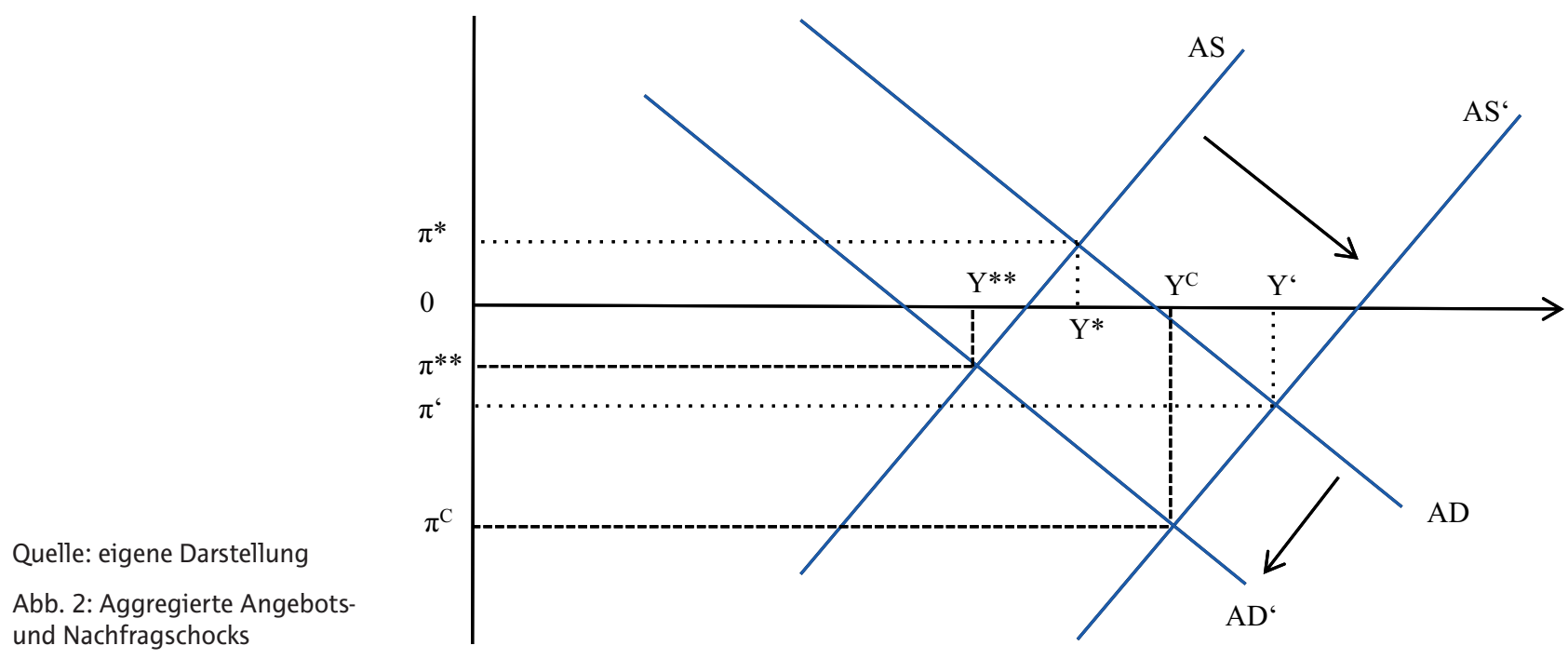




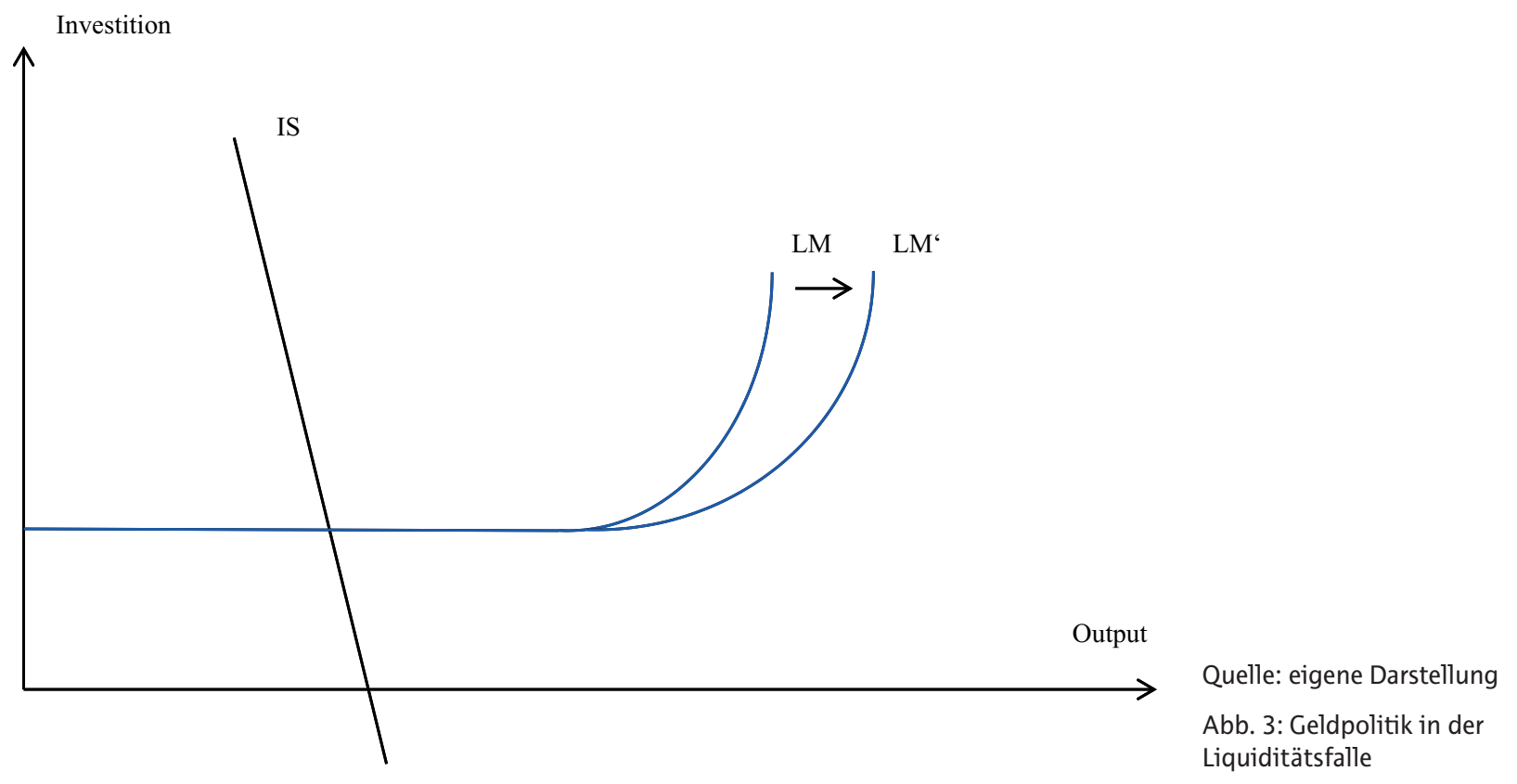

nehmer nicht von einer zukünftigen Änderung der Geldpolitik ausgehen, werden sie auf Erhöhungen des Geldangebots nicht reagieren, da sie diese als lediglich temporär erachten. Die grundlegende Herausforderung der Geldpolitik in einem deflationären Umfeld liegt also darin, den Marktteilnehmern einen dauerhaften (statt vorübergehenden) Anstieg des Preisniveaus glaubhaft zu versichern (vgl. Krugman, 1998, S. 194 ff. und Eggertsson, Woodford, 2003, S. 139 ff.)

Die Bedeutung der Erwartungen ist für die Beständigkeit der Deflation von kritischer Bedeutung. Annahmegemäß sei der heutige Güterpreis $\mathrm{P}$ relativ zu einem zukünftigen Güterpreis $\mathrm{P}^{\mathrm{e}}$, also das Verhältnis $\mathrm{P} / \mathrm{P}^{\mathrm{e}}$, zu hoch, was dazu führt, dass Verbraucher ihre heutigen Ausgaben zugunsten zukünftiger Ausgaben reduzieren (Konsumzurückhaltung). Gesamtwirtschaftlich betrachtet führt dies dazu, dass es in der laufenden Periode zu einem Überschuss an Sparen zu Lasten des Investierens käme. Ein Marktgleichgewicht ließe sich durch ein Absenken von P oder eine Erhöhung von $\mathrm{P}^{\mathrm{e}}$ erreichen. Sobald das aktuelle Preisniveau jedoch zu sinken beginnt, wird auch das zukünftig erwartete Preisniveau sinken, was die gesamtwirtschaftliche Aktivität insgesamt weiter verlangsamen wird und in letzter Instanz die Output-Inflations-Dynamik zu verändern vermag. Unter Umständen könnte es zu einer Abflachung der Phillips-Kurve kommen, was im Ergebnis dazu führt, dass der aggregierte Nachfrageschock zu einem starken Rückgang von Output und Beschäftigung führt, der anfangs kaum Einfluss auf die Preise hat. Eine langfristige Schwäche von Output und Beschäftigung hingegen schlägt sich in der Regel in zunehmendem Druck auf die Preise nieder. Dies kann unter Umständen zu einer deflationären Spirale, die darauf zurückgeht, dass heute fallende Preise die Erwartung auch zukünftig fallender Preise verstärken, führen.

\section{Deflation in Japan - Die verlorene Dekade}

Abgesehen von einer kurzen Unterbrechung Ende der 1990er Jahre verzeichnete die in Japan beobachtete Preisentwicklung, gemessen als Veränderung des BIP-Deflators gegenüber dem Vorjahr, seit 1992 einen kontinuierlichen Rückgang. Infolge einer expansiven Geldpolitik der Bank of Japan (BoJ), die neben einer Verdopplung der Geldmenge M0 (d. h. der Geldbasis, die sich aus dem im Umlauf befindlichen Bargeld sowie den Einlagen der Geschäftsbanken bei der BoJ zusammensetzt) bis Ende 2014 auch den (unbefristeten) Kauf japanischer Staatsanleihen mit einer Laufzeit von bis zu 40 Jahren sowie den Kauf börsennotierter Indexfonds (ETFs) und Immobilienfonds (REITs) umfasst, ist seit Mitte 2013 ein moderater Preisanstieg zu beobachten. Nach 1,2\% p.a. im November 2013 stieg der japanische Verbrauchpreisindex (ex Lebensmittel) im Dezember 2013 um 1,2\% p.a., dem höchsten Anstieg seit den im Oktober 2008 beobachteten 1,9\% p.a. Dieser ging mit einer Erhöhung der Industrieproduktion in Höhe von $1,1 \%$ p.a. im Dezember 2013 einher. Im Gesamtjahr 2013 stieg der japanische Verbraucherpreisindex um 0,4\% p.a.; dem ersten Anstieg in den vergangenen fünf Jahren (vgl. Dey, 2014). Inflationsprognosen der BoJ gehen aktuell von einer Inflationsrate in Höhe von $0,5 \%$ bis $0,8 \%$ p.a. für das im März endende Fiskaljahr 2014 und von 1,9\% p.a. im Fiskaljahr 2015 aus; Werte die der Internationale Währungsfonds (IMF) jedoch in Frage stellt (vgl. BoJ, 2014 und IMF, 2014).

\section{Gründe für die Deflation}

Gefolgt von mehreren Quartalen eines fallenden BIP-Deflators fiel die Kerninflationsrate in Japan bereits Mitte der 1980er-Jahre auf Null. Nachdem sich Ende 1987 ein starker Anstieg des wirtschaftlichen Wachstums abzeichnete, welcher mit der zeitgleichen Erholung der Vermögenspreise, allen voran der Immobilien- und Aktienpreise, einherging, kam es sukzessive zu einer Überhitzung der 
Volkswirtschaft, deren Wachstumsrate Anfang der 1990er Jahre zwischen zwei und drei Prozentpunkten über ihrem Potenzialwachstum stand. Der Verbraucherpreisindex hingehen nahm nur moderat zu und begann nach dem Erreichen eines Höhepunktes von $3 \%$ p.a. infolge einer zunehmend restriktiven Geldpolitik der BoJ, welche zudem eine Korrektur der Vermögenspreise nach sich zog, bereits Anfang 1991 zu fallen.Zur Veranschaulichung: während der japanische Aktienindex Nikkei 225 sich in den vier Jahren von 1985 bis 1989 von knapp 13.000 auf über 38.000 Punkte verdreifachte, fiel er zwischen 1990 und 1992 auf nur noch 16.000 Punkte zurück.

Diese Entwicklung, bzw. der sich andeutende Abschwung des Konjunkturzyklus, veranlasste japanische Konsumenten zu einem wesentlich stärkeren Sparverhalten als zuvor. Die daraus resultierende Konsumzurückhaltung trug zu einer weiteren Unterauslastung der Produktionskapazitäten bei. Infolge dieser Entwicklung, die sich in rückläufigen Umsätzen und Gewinnen niederschlug, kam es seitens der betroffenen Unternehmen zu einer Investitionszurückhaltung. In der Summe führte diese Zurückhaltung zu einem Rückgang der Gesamtgüternachfrage bei kurzfristig gleichbleibendem Güterangebot; d. h. es entstand eine sogenannte Nachfragelücke.

\section{a) Wachsende Output-Lücke lastete auf Preisniveau}

Der abrupte Rückgang der Vermögenspreise Anfang der 1990er-Jahre bewirkte zunächst einen Stillstand des realen BIP-Wachstums. Die sich insbesondere aus der in der Fertigungs- und Bauindustrie entwickelnden Überschusskapazität entstehende Output-Lücke führte zu weiterem Druck auf das Preisniveau. Banken, die noch in den Vorjahren zahlreiche Kredite an den Bau- und Immobiliensektor vergeben hatten, sahen sich mit einem abrupten Anstieg notleidender Kredite sowie einer abnehmenden Profitabilität konfrontiert. Infolge der Konsolidierung ihrer Bilanzen kam es zu einem spürbaren Rückgang der Vergabe neuer Kredite; das Wachstum des Geldmengenaggregats M2, beispielsweise, fiel von über $11 \%$ p.a. 1990 auf nur noch 0,6\% p.a. 1992.

\section{b) Spill-Over Effekte seitens Externer Schocks}

Japan sah sich in den vergangenen Jahrzehnten mit einer Vielzahl verschiedener Krisen konfrontiert. Auf das Platzen der Spekulationsblase Anfang der 1990-er Jahre folgte zunächst die Korrektur der US-Aktienmärkte, der Kollaps der US-Investmentbank Lehman Brothers 2008 sowie das Erdbeben in der Region Tohoku 2011. Während dieser Phase fielen die Notierungen japanischer Aktien um $80 \%$ und verharren, analog zu den Immobilienpreisen, aktuell knapp $60 \%$ unterhalb der 1989 bzw. 1991 beobachteten Höchststände.

Zwar kam es nach jedem einzelnen dieser Schocks zu einer zaghaften wirtschaftlichen Erholung, dennoch machten die kurz darauffolgenden Schocks Japans Volkswirtschaft infolge einer wachsenden Output-Lücke sukzessive anfälliger für eine Deflation.

\section{c) Inadäquate Reaktion seitens der Politik}

Nach Maßgabe der Literatur (vgl. Bernanke, Gertler, 2001, S. 253 ff.) reagierte die BoJ verspätet und in nicht ausreichendem Maße auf die wirtschaftliche Entwicklung Japans, d. h. sowohl die Straffung der Geldpolitik als Reaktion auf das spekulative Wachstum Ende der 1980-er Jahre wie auch die Lockerung der Geldpolitik nach dem Platzen der spekulativen Blase und der nachfolgenden Verlangsamung des wirtschaftlichen Wachstums erfolgten zu spät. So deutet eine simple Taylor-Regel zumindest ex post darauf hin, dass die japanische Geldpolitik in Anbetracht der wirtschaftlichen Lage seit 1998 kontinuierlich zu restriktiv war; mittlerweile jedoch fast zu akkommodierend erscheint. Die erste Zinssenkung erfolgte damals erst mehr als 18 Monate nach dem Platzen der spekulativen Blase und es sollte bis 1999 dauern, bis der Hauptrefinanzierungssatz nach mehreren aggressiven Zinssenkungen offiziell auf Null gesenkt wurde. Zudem kam es erst im März 2001 zu einem Quantitative Easing, d. h. einer Ausweitung der Bilanzsumme der BoJ durch den Ankauf von Staatsanleihen, welche allerdings weit hinter den Reaktionen der führenden Zentralbanken auf die Insolvenz von Lehman Brothers im September 2008 zurückblieb.

Gleiches galt für die Fiskalpolitik der japanischen Regierung: infolge der verspäteten Intervention in Form des deficit spending erfolgte keine nennenswerte Verbesserung der Lage. Der Einfluss der Geld- und Fiskalpolitik erwies sich als begrenzt und der Leitzins der BoJ verharrt seit Jahren nahe bzw. bei Null, ohne dass dies bisher zu einer spürbaren Erholung der Volks-wirtschaft geführt hätte.

\section{d) Entwicklung der Inflationserwartungen}

Aktuelle Studien der BoJ (vgl. Nishizaki et al., 2012, S. 1 ff.) deuten darauf hin, dass es seitens der Verbraucher bereits Mitte der 1990-er Jahre zu einer Anpassung der kurzfristigen Inflationserwartungen kam. Während die Verbraucher zunächst davon ausgingen, dass der Rückgang der Inflation insgesamt lediglich temporär sei, erfolgte ab 1996 eine Entkopplung der kurz- und langfristigen Inflationserwartungen. So sanken die kurzfristigen, d. h. die einjährigen Inflationserwartungen erstmals 1998 unter Null, doch sollte es bis 2003, d. h. fünf Jahre nach dem ersten Auftreten der Deflation, dauern, bis die Verbraucher auch dauerhaft von einer Inflationsrate von unter Null ausgingen.

\section{e) Makroökonomische Entwicklung}

Aufgrund des zunehmenden Markteintritts chinesischer Exporteure sowie der weiteren wirtschaftlichen Öffnung Japans sowie dem damit einhergehenden Abbau von Handelshemmnissen, insbesondere gegenüber der Volksrepublik China, kam es nicht zuletzt infolge komparativer Wettbewerbsnachteile seit den 1980-er Jahren zu weiterem Druck auf die Preise von Handelsgütern. Die entsprechende Verschärfung des Wettbewerbs lässt sich an der Entwicklung der jeweiligen Marktanteile beobachten, die darauf hindeuten, dass japanische Exporteure ungeachtet 


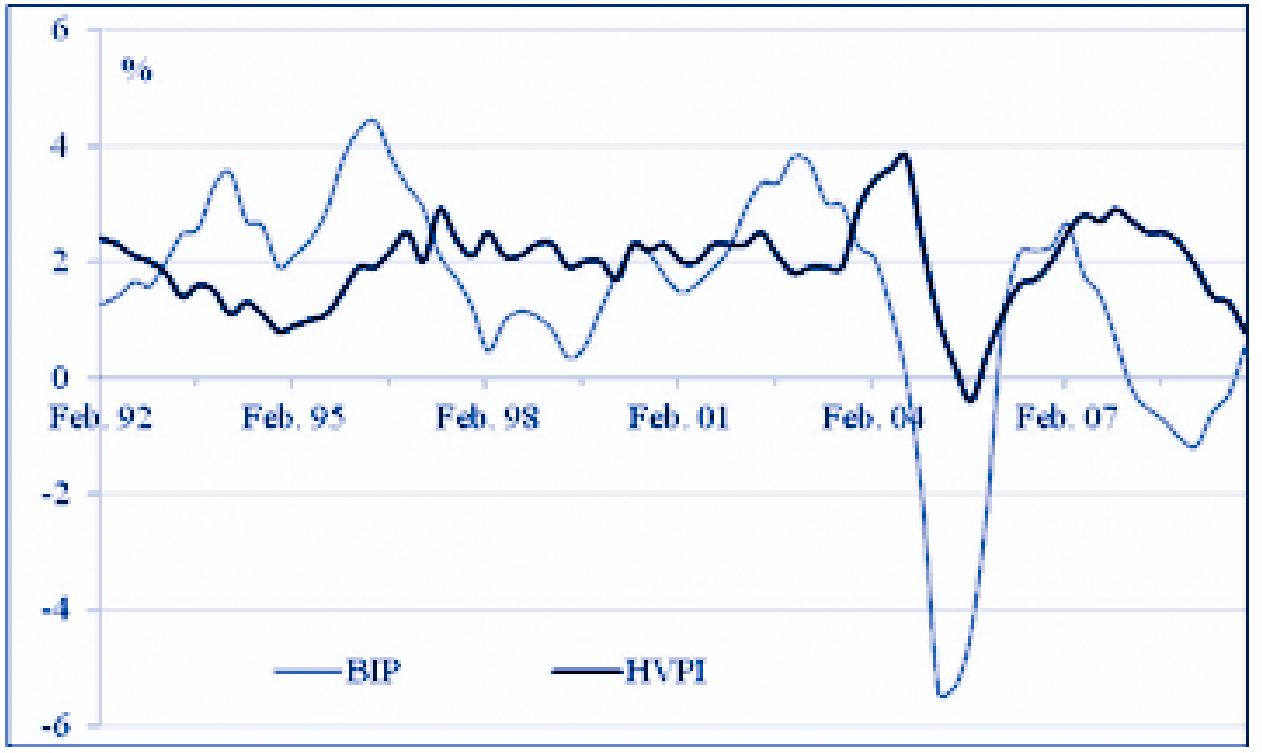

Quelle: Eurostat (2014)

Abb. 4: Entwicklung (in \%) des BIP und HICP in der EWU (Q1 1996 bis Q4 2013) deutlicher Preissenkungen im vergangenen Jahrzehnt spürbare Einbußen hinnehmen mussten.

\section{Die Lage in der Eurozone}

Insbesondere infolge sinkender Rohölpreise fiel die Inflationsrate (HICP) in der EWU nach noch 0,9\% im November 2013 auf lediglich 0,3\% p.a. im November 2014 zurück (vgl. Abb. 4). Für das Gesamtjahr 2014 erwartet die EZB eine Inflation in Höhe von 1,1\%. Damit blieb sie deutlich hinter dem avisierten Zielwert von ,unter aber nahe“ 2,00 \% p.a. zurück, der letztmals im Januar 2013 erreicht wurde (vgl. EZB, 2014). Und auch wenn es im vierten Quartal 2013 zu einer leichten Erholung des aggregierten Bruttoinlandsproduktes (BIP) der EWU-Mitgliedsstaaten kam, sollte ein Wachstum von nur 0,5\% p.a. nicht darüber hinwegtäuschen, dass die europa-weite wirtschaftliche Erholung sich als ausgesprochen verletzlich erweist; insbesondere in Anbetracht eines durchgängig negativen jährlichen Wachstums, d. h. einer Rezession, in den sieben Quartalen von Q1 2012 bis Q3 2013. Für das Jahr 2014 erwartetet die EZB ein Wirtschaftswachstum in Höhe von 1,00\% p.a. für die EWU (vgl. EZB, 2014).

\subsection{Gemeinsamkeiten mit Japan}

\section{a) Ausmaß der Korrektur der Märkte}

Selbst wenn das Ausmaß der Korrektur der Aktienmärkte über den gesamten Krisenzeitraum hinweg betrachtet in Japan stärker ausgefallen ist, zeichnen sich frappierende Ähnlichkeiten zwischen der Entwicklung in Japan seit 1989 und der Eurozone seit 2007 ab. Mit einem Kursrückgang von mehr als $60 \%$ in den 18 Monaten seit Ausbruch der Krise 2007 fiel die Korrektur der Aktienmärkte in der Eurozone sogar stärker aus als in dem vergleichbaren Zeitraum in Japan. Eine deutliche Korrektur der Immobilienpreise hingegen lässt sich nicht beobachten; eine Entwicklung, die nicht zuletzt auf den Preisan- stieg deutscher Immobilien zurückzuführen ist. Hervorzuheben ist also, sollte die Deflation der Preise für Anlagegüter ein verlässlicher Früh-indikator für eine bevorstehende Deflation sein, dass ein für die Eurozone durchaus alarmierendes Signal gegeben scheint.

Dennoch ist der Tatsache Rechnung zu tragen, dass während sich Japan in den 1990-er Jahren de facto orientierungslos Richtung Deflation bewegte, die Eurozone sich in der vergleichsweise bequemen Lage befindet, aus den damals gesammelten Erfahrungen lernen zu können.

\section{b) Die Rolle der Geldpolitik}

Nach einer im Verlauf der Krise zumeist adäquaten geldpolitischen Maßnahme fällt die Geldpolitik der EZB nach Maßgabe einer einfachen Taylor-Regel in Anbetracht der zuletzt stockenden wirtschaftlichen Erholung der Eurozone mittlerweile vergleichsweise restriktiv aus; eine Entwicklung, die u. a. durch die aktuelle Verringerung der Bilanzsumme der EZB beschleunigt wird und deutliche Parallelen zu jener in Japan aufweist. Gleichwohl stellt sich in Anbetracht des historisch niedrigen Hauptrefinanzierungszinssatzes in Höhe von aktuell nur noch $0,05 \%$ und einer bisher bestenfalls verhaltenen wirtschaftlichen Erholung in (zahlreichen Mitgliedsstaaten) der EWU die Frage nach der Wirksamkeit weiterer geldpolitischer Schritte; insbesondere nach dem Sinn einer weiteren möglichen Zinssenkung und der Bedeutung des Zinskanals (siehe auch $A b b$. 3).

\section{c) Die Bedeutung des Bankensektors}

Während der Abbau notleidender Kredite seitens japanischer Geschäftsbanken infolge des mangelnden politischen Drucks erst relativ spät und selbst dann eher zögerlich erfolgte, ist in der Eurozone ein frühes und entschiedenes Vorgehen zur Re-Kapitalisierung des Bankensektors, die noch immer nicht abgeschlossen ist, zu beobachten. Da es des Weiteren kaum mehr wechselseitige Eigen- 
kapitalbeteiligungen der Banken der Eurozone gibt, erwiesen sich die auf die Finanzkrise 2007 zurückzuführenden Ansteckungseffekte als weit weniger stark ausgeprägt als in Japan. Anlass zur Sorge bereitet gleichwohl der spürbare Rückgang der Kreditvergabe in der Eurozone (vgl. Dierks, 2013, S. 10 ff.).

\section{d) Verankerung der Inflationserwartungen}

Ungeachtet obiger Entwicklungen geht die EZB gegenwärtig nicht davon aus, dass die EWU aus einer Phase aktuell niedriger Inflation, die unter Umständen auch länger Bestand haben könnte, in eine Periode nachhaltiger Deflation abrutschen wird. Dies ist insbesondere darauf zurückzuführen, dass die langfristigen Inflationserwartungen der Finanzmarktakteure fest , unter aber nahe“ 2,00 \% p.a. verankert sind und das Wirtschaftswachstum der Region sich sukzessive erholen wird (vgl. EZB, 2014). Dennoch mehren sich Stimmen (vgl. Kennedy, 2014), die darauf hinweisen, dass die EZB die langfristige Entwicklung der Inflationsrate systematisch überschätzt, nicht zuletzt deshalb, da die kurzfristigen Inflationserwartungen seitens der Verbraucher, wie schon 1998 in Japan, bereits spürbar gesunken sind. Ein weiteres Absenken des Hauptrefinanzierungszinssatzes auf Null, beispielsweise, könnte die kurzfristigen Inflationserwartungen weiter drücken - und damit den deflationären Tendenzen in der Eurozone weiter Vorschub leisten.

\subsection{Unterschiede zu Japan}

\section{a) Die Eurozone als Gemeinschaft von Märkten}

Auch wenn eine ähnliche Geldpolitik als Determinante deflationärer Tendenzen den Vergleich zwischen Japan und der Eurozone geradezu aufnötigt, sind im Falle der Eurozone die heterogenen Ausprägungen der verschiedenen Volkswirtschaften und ihrer Finanzmärkte hervorzuheben. Dies ist insbesondere deshalb bedeutsam, da es im Vorfeld der gegenwärtigen Staatsschuldenkrise innerhalb der Europäischen Währungsunion zu einem mehrjährigen Aufbau interner Ungleichgewichte kam. Naturgemäß macht der anhaltende Abbau dieser Ungleichgewichte bei einer für die Eurozone einheitlichen Geldpolitik seitens der EZB einige Volkswirtschaften verletzlicher für eine Deflation als andere: im jetzigen Marktumfeld, d. h. bei einem Hauptfinanzierungszinssatz in Höhe von 0,05\%, könnte sich eine für Deutschland adäquate Geldpolitik für Spanien, beispielsweise, als unter Umständen zu restriktiv erweisen.

\section{b) Die Rolle der Fiskalpolitik}

Ein weiterer, wesentlicher Unterschied zwischen der damaligen Lage in Japan und der heutigen Situation in der Eurozone ist in der Fiskalpolitik zu sehen. Während in Japan von Anfang an eine expansive Fiskalpolitik, das sogenannte deficit spending, im Zentrum der Krisenbewältigung stand, die dazu führte, dass Japan mit mittlerweile $245 \%$ des BIP (Stand: Ende 2013) die am stärksten verschuldete Industrienation der Welt ist, fiel die Antwort der
Mitgliedsstaaten der Eurozone grundverschieden aus. So kam es im Vorfeld der jetzigen Staatsschuldenkrise, insbesondere im Falle der Mittelmeeranrainer, zwar zu einer vergleichsweise expansiven Fiskalpolitik, doch wurde diese in jüngster Vergangenheit durch einen europaweit deutlichen Rückgang der Staatsausgaben kompensiert, der, ceteris paribus, dazu führt, dass die strukturellen Defizite der Volkswirtschaften der Eurozone bis Ende des Jahrzehnts abgebaut werden. Gleichwohl darf bei dieser Betrachtung nicht außer Acht gelassen werden, dass die fiskalische Austerität nicht ohne Auswirkungen auf die Output-Lücke bleibt, was, insbesondere im Falle der Mittelmeeranrainer, die deflationären Tendenzen weiter verstärken dürfte.

\section{c) Deflation als Teil der Lösung?}

Ein weiterer Unterschied zwischen der damaligen Lage in Japan und der aktuellen Situation in der Eurozone liegt darin, dass eine zeitweise Deflation innerhalb der Eurozone zur Lösung der Staatsschuldenkrise unter Umständen unabdingbar ist. Da es aufgrund der Währungsunion und der damit einhergehenden Gemeinschaftswährung innerhalb der EWU zu keiner Auf- bzw. Abwertung der Wechselkurse kommen kann, ist eine interne Abwertung für zahlreiche Mitgliedsstaaten eine willkommene Möglichkeit, ihre Wettbewerbsfähigkeit wieder herzustellen.

Das im vorigen Konjunkturzyklus in mehreren Volkswirtschaften der Eurozone insbesondere durch eine exzessive Kreditvergabe geprägte Wirtschaftswachstum führte u. a. dazu, dass Fundamentaldaten wie beispielsweise die Effizienz der Arbeits- oder Gütermärkte nicht hinreichend beachtet wurden. Sofern die Kosten des Faktors Arbeit jedoch schneller als die Produktivität wachsen, kommt es zu einer Zunahme der Lohnstückkosten, was sich im Zeitraum seit der Gründung der EWU bis zum Ausbruch der Krise 2007 empirisch vor allem in den peripheren Mitgliedsstaaten beobachten ließ.

Die infolge der Krise verabschiedeten Reformmaßnahmen, so z. B. moderate Lohnabschlüsse und Preiserhöhungen sowie die steuerliche Verbilligung des Faktors Arbeit zu Lasten des Konsums, führten dahingehend zu einer internen Abwertung, dass Lohnstückkosten und Produktivität wieder im Einklang waren. Dies verbessert die Wettbewerbsfähigkeit und reduziert dergestalt die entstandenen Leistungsbilanzdefizite.

\section{Fazit: Kurzfristig erscheint eine Deflation nicht unmöglich}

In Anbetracht mehrerer, z. T. frappierender Ähnlichkeiten zu der volkswirtschaftlichen Dynamik Japans während des Verlorenen Jahrzehnts scheinen sich Befürchtungen einer der Eurozone bevorstehenden Deflation zu bewahrheiten. In Mitgliedsstaaten wie aktuell z. B. Spanien oder Griechenland, wo sich der Rückgang des Preisniveaus 2013 auf $0,9 \%$ p.a. belief, ist dieses Phänomen bereits zu beobachten. 


\begin{tabular}{|l|l|l|l|}
\hline Ursache & \multicolumn{1}{|c|}{$\begin{array}{c}\text { Einfluss auf } \\
\text { Preisniveau }\end{array}$} & \multicolumn{1}{c|}{$\begin{array}{c}\text { Japan des } \\
\text { Verlorenen Jahrzehnts }\end{array}$} & \multicolumn{1}{c|}{ Eurozone heute } \\
\hline Externer Schock & deutlich negativ & starke, frühe Determinante & mäßige, frühe Determinante \\
\hline Fiskalpolitik & mäßig negativ & $\begin{array}{l}\text { expansiv; begünstigt } \\
\text { Inflation }\end{array}$ & $\begin{array}{l}\text { restriktiv, begünstigt } \\
\text { Deflation }\end{array}$ \\
\hline Geldpolitik & deutlich negativ & $\begin{array}{l}\text { starker Einfluss, besonders } \\
\text { nach Eintreten in Deflation }\end{array}$ & aktuell bereits relativ eng \\
\hline $\begin{array}{l}\text { Inflations- } \\
\text { erwartungen }\end{array}$ & mäßig negativ & $\begin{array}{l}\text { mäßiger Einfluss nach } \\
\text { Eintritt in Deflation }\end{array}$ & $\begin{array}{l}\text { kurzfristige Erwartungen } \\
\text { sinken }\end{array}$ \\
\hline
\end{tabular}

Quelle: eigene Darstellung

Abb. 5: Determinanten der Deflation - Japan und die Eurozone
Doch ungeachtet zahlreicher Parallelen wie z. B. der Rolle der Geldpolitik oder der Verankerung der Inflationserwartungen, überwiegen die Unterschiede; allen voran die Tatsache, dass die Eurozone eine zutiefst heterogene Gemeinschaft verschiedener Volkswirtschaften und Finanzmärkte ist. So ist aktuell nur bedingt davon auszugehen, dass die Eurozone aus einer Periode aktuell niedriger Inflation, die unter Umständen auch noch länger Bestand haben könnte, in eine Periode anhaltender Deflation abrutschen wird.

Als entscheidend für die weitere Entwicklung dürfte sich 2015 erweisen: sollten sich die bislang sehr verhaltenen Inflationsraten in größeren Volkswirtschaften wie Deutschland (0,7\% p.a. im Oktober 2014), Frankreich $(0,5 \%)$ oder Italien $(0,2 \%)$ im weiteren Verlauf in deflationäre Tendenzen umkehren, so könnte die Eurozone $(0,4 \%)$ insgesamt sehr wohl eine Deflation erfahren. Nicht zuletzt aufgrund der Heterogenität der wirtschaftlichen Entwicklung innerhalb der Eurozone dürfte sich diese jedoch als bestenfalls vorübergehend erweisen.

\section{Literatur}

Bank of Japan, Monthly Report of Recent Economic and Financial Developments March 2014, Tokio.
Bernanke, B., M. Gertler, Should Central Banks Respond to Movements in Asset Prices?, in: American Economic Review, Jg. 91(2), S. 253-257.

Dey, $S$., Risk of deflation in euro zone seen by economists as more serious than ECB says: poll, 2014, URL: http://uk.reuters.com/ article/2014/02/13/us-economy-eurozone-poll-idUKBREA1C1 DT20140213 (Abrufdatum: 28.03.2014).

Dierks, L., Ein Paradoxon: Wie niedrige Zinsen die Refinanzierung verteuern, in: Bond Yearbook 2013/14, November 2013, S. 10-14.

Eggertsson, G., M. Woodford, The Zero Bound on interest Rates and Optimal Monetary Depression, in Brookings Papers on Economic Activity I, (2003), S. 139-233.

Europäische Zentralbank, Monatsbericht der Europäischen Zentralbank, März 2014.

International Monetary Fund, Deflation: Determinants, Risks, and Policy Options - Findings of an Interdepartamental Task Force, Washington, 2003.

International Monetary Fund, World Economic Outlook 2014 - Is the Tide Rising?, Washington, 2014.

Kennedy, S., ECB May Repeat Japan Mistake That Triggered Lost Decade, 2014, URL: http://www.bloomberg.com/news/201403-05/ecb-may-repeat-japan-mistake-that-triggered-lost-decade. html (Abrufdatum: 28.03.2014).

Krugman, P., It baaack: Japan's Slump and the Return of the Liquidity Trap, in: Brookings Papers on Economic Activity II, (1998), S. 194-199.

Nishizakli, K., T. Sekine, Y. Ueno, Chronic deflation in Japan, Bank of Japan Working Paper Series, Juli 2012

\section{WiSt}

Schriftleitung: Verantwortliche Redakteure: für Betriebswirtschaftslehre Prof. Dr. Michael Lingenfelder, Universität Marschaftslehre Prof. Dr. Michael Lingenfelder, Universität Mar-
burg, FB02, BWL III, Universitätsstraße 24, 35032 Marburg, burg, FB02, BWL 11 , Universiatatsstraße 24, 35032 Marburg, Telefon: 06421/282 37 63; für Volkswirtschaftslehre Prof. Dr. Norbert Berthold, Universität Würzburg, Sanderring 2,
97070 Würzburg, Telefon: 0931/3 18 2925. Mitarbeiter: Dipl.97070 Würzburg, Telefon: 0931/3 182925. Mitarbeiter: Dipl.marburg.de, Dipl.-Volksw. Jörg Rieger, Würzburg, E-Mail joerg.rieger@uni-wuerzburg.de.

Manuskripte: Manuskripte sind an die Redaktion zu senManuskripte: Manuskripte sind an die Redaktion zu senden. Der Verlag haftet nicht fur Manuskripte, die unverlangt eingereicht werden. Sie können nur zurückgegeben werden, wenn Rückporto beigefügt ist. Die Annahme zur Veröffentlichung muss schriftlich erfolgen. Mit der Annahme zur Veröffentlichung überträgt der Autor dem Verlag C.H.BECK an seinem Beitrag für die Dauer des gesetzlichen Urheberrechts das exklusive, räumlich und zeitlich unbeschränkte Recht zur Vervielfältigung und Verbreitung in körperlicher Form, das Recht zur öffentlichen Wiedergabe und Zugänglichmachung, das Recht zur Aufnahme in Datenbanken, das Recht zur Speicherung auf elektronischen Datenträgern und das Recht zu deren Verbreitung und Vervielfältigung sowie das Recht zur sonstigen Verwertung in elektronischer Form. Hierzu zählen auch heute noch nicht bekannte Nutzungsformen. Das in $\$ 38$ Abs. 4 noch nicht bekannte Nutzungsformen. Das in $\$ 38$ Abs. 4 UrhG niedergelegte zwingende Zweitverwertungsrecht de Autors nach Ablauf von $12 \mathrm{M}$

Urheber- und Verlagsrechte: Alle in dieser Zeitschrift veröffentlichten Beiträge sind urheberrechtlich geschützt.
Das gilt auch für die veröffentlichten Gerichtsentscheidungen und ihre Leitsätze, denn diese sind geschützt, soweit sie vom Einsender oder von der Schriftleitung erarbeitet oder redigiert worden sind. Der Rechtsschutz gilt auch gegenüber Datenbanken und ähnlichen Einrichtungen. Kein Teil dieser Zeitschrift darf außerhalb der engen gen. Kein Teil dieser Zeitschrift darf außerhalb der enge-
Grenzen des Urheberrechtsgesetzes ohne schriftliche GeGrenzen des Urheberrechtsgesetzes ohne schriftliche Ge-
nehmigung des Verlags in irgendeiner Form vervielfälnehmigung des Verlags in irgendeiner Form vervielfäl-
tigt, verbreitet oder öffentlich wiedergegeben oder zugänglich gemacht, in Datenbanken aufgenommen, au elektronischen Datenträgern gespeichert oder in sonstige Weise elektronisch vervielfältigt, verbreitet oder verwertet werden.

Anzeigenabteilung: Verlag C.H.BECK, Anzeigenabteilung, Wilhelmstraße 9, 80801 München, Postanschrift: Postfach 4003 40, 80703 München

Media-Beratung: Telefon 089/3 81 89-687, Telefax 089/ $38189-589$.

Disposition, Herstellung Anzeigen, technische Daten Telefon 089/3 81 89-603, Telefax 089/3 81 89-589, E-Mai anzeigen@beck.de

Verantwortlich für den Anzeigenteil: Bertram Götz

Verlag: C.H.BECK oHG, Wilhelmstraße 9, 80801 München, Telefon: 089/381 89-0, Telex: 5215085 beck d. Telefax: 089/38 1893 98, Postbank: München, Kto. 6229-802, BLZ 700100 80. Der Verlag ist oHG. Gesellschafter sin Dr. Hans Dieter Beck und Dr. h. c. Wolfgang Beck, beide Verleger in München.

Erscheinungsweise: Monatlich

Bezugspreise 2015: Halbj. € 99,- (darin € 7,50 MwSt.), Jahrestitelei und -register sind nur noch mit dem jeweiligen Heft lieferbar.
Vorzugspreis für Studenten (fachbezogener Studiengang, gegen Nachweis) € 49,- (darin € 3,71 MwSt.).

Kombipreis (inkl. Campuslizenz) $€ 132,-($ darin $€ 10$,MwSt.)

Die Abopreise sind inklusive WiSt-Archiv online für einen Arbeitsplatz.

Einzelheft: $€ 19,50$ (darin $€ 1,28$ MwSt.) jeweils zuzüglich Versandkosten.

Nicht eingegegangene Exemplare können nur innerhalb von 6 Wochen nach dem Erscheinungstermin reklamiert werden.

Bestellungen nehmen entgegen: jede Buchhandlung und der Verlag.

KundenServiceCenter: Tel.: 089/381 89-750. Fax: 089/ 381 89-358. E-Mail: bestellung@ beck.de.

Abbestellungen müssen 6 Wochen vor Halbjahresschluss erfolgen.

Adressenänderungen: Teilen Sie uns rechtzeitig Ihre Adressenänderungen mit. Dabei geben Sie bitte neben dem Titel der Zeitschrift die neue und die alte Adresse an. Hinweis gemäß $§ 7$ Abs. 5 der Postdienst-Datenschutzverordnung:

Bei Anschriftenänderung des Beziehers kann die Deutsche Post AG dem Verlag die neue Anschrift auch dann mitteilen, wenn kein Nachsendeantrag gestellt ist. Hiergegen kann der Bezieher innerhalb von 14 Tagen nach Erscheinen dieses Heftes beim Verlag widersprechen.

Satz: FotoSatz Pfeifer GmbH, 82166 Gräfelfing.

Druck: Druckerei C.H.Beck, Bergerstr. 3, 86720 Nördlingen. 